\title{
HYPER-AFL'S AND ETOL SYSTEMS
}

P. A. Christensen

Department of Computer Science

University of Aarhus

Aarhus, Denmark

The notions of K-iteration grammars and of hyper-AFL's are introduced in $[57]$ and [103]. The notation follows that of [1 03].

\section{Theorem}

$$
E T O L=E T O L(1)=E T O L_{i t e r}, \text { i.e. ETOL is a hyper-AFL. }
$$

\section{Sketch of a proof}

It is obvious from the definition of an iteration grammar that

ETOL $\subseteq$ ETOL $_{\text {ter }}^{(1)} \subseteq$ ETOL $_{\text {iter }}$.

To prove ETOL $i$ ter $\subseteq$ ETOL, let $G=\left(V_{N}, V_{T}, S, U\right)$ be an ETOL-iteration grammar with $u=\left\{\mathcal{T}_{1}, \ldots, \mathcal{T}_{n}\right\}$, where each $\mathcal{T}_{\mathbf{j}}$ is an ETOL-substitution.

Assume that $\mathcal{T}_{j}\left(a_{i}\right)=L\left(G_{i, j}\right)$, where $G_{i, j}=\left(V_{N}^{i, j}, V_{T} \cup V_{N}, T_{i, j}, s_{i, j}\right)$ are synchronized versions of ETOL-systems and the alphabets $V_{N}^{l, j}$ are pairwise disjoint.

We define a new ETOL-system: $G^{\prime}=\left(V_{N}^{\prime}, V_{T}^{\prime}, T^{\prime}, \overline{\overline{5}}\right)$, where

$V_{N}^{\prime}=\{\$\} \bigcup_{i, j}\left(V_{N}^{i, j} \cup\left\{\bar{S}_{i, j}\right\}\right) \cup \bar{V}_{T} \cup \bar{V}_{N} \cup \bar{V}_{T} \cup \bar{\nabla}_{N}$ where $\$$ and all $\bar{s}_{i, j}$ are new symbols.

$\bar{V}_{X}=\left\{\bar{a} \mid a \in V_{X}\right\}$ and $\bar{\nabla}_{X}=\left\{\bar{a} \mid a \in V_{X}\right\}$ for $x=N$ and $X=T$ are sets of new symbols.

If $x$ is a string of symbols $x=b_{1} \ldots b_{k}$, then $\bar{x}=\bar{b}_{1} \ldots \bar{b}_{k}$ and $\overline{\bar{x}}=\bar{b}_{1} \ldots \bar{b}_{m}$. The axiom of $G^{\prime}$ is defined as $\overline{\bar{s}}$ in exactly the same way.

Finally $T^{\prime}$ consists of the tables:

$\overline{\bar{a}}_{i} \rightarrow \bar{s}_{1, j}$ for each $i$ and each $j$

$t_{0}:$

$A \rightarrow \$$ for any other symbol A.

For $1 \leq j \leq n$ there is the table: 
$\bar{s}_{i, j} \rightarrow \bar{s}_{i, j} ; \bar{s}_{i, j} \rightarrow s_{i, j}$ for each $i$

$t_{j}:$

$\overline{\mathrm{a}} \rightarrow \overline{\bar{a}} \quad ; \overline{\bar{a}} \rightarrow \overline{\bar{a}} \quad$ for each $a \in V_{N} \cup V_{T}$

$A \rightarrow \$$ for any other symbol $A$.

For $1 \leq j \leq n$ and $1 \leq i \leq\left|V_{N} \cup V_{T}\right|$ there is the set of tables:

which consists of all tables from $T_{i, j}$

$\widetilde{T}_{i, j}$ : where the table with the terminal productions $\left(G_{i, j}\right.$ is synchronized $)$ is changed to produce barred terminals instead. In all these tables we add the productions:

$$
\begin{array}{ll}
\overline{\bar{a}} \rightarrow \overline{\bar{a}} & \text { for each } a \in V_{N} \cup V_{T} \\
\bar{S}_{k, j}+\bar{S}_{k, j} & \text { for each } k \\
A \rightarrow \$ & \text { for any other new symbol. }
\end{array}
$$

Finally there is the table with the terminal productions:

$$
\begin{aligned}
& \bar{a} \rightarrow \text { a for each } a \in V_{T} \\
& A \rightarrow \$ \text { for any other symbol } A .
\end{aligned}
$$

The claim is now that $L(G)=L\left(G^{\prime}\right)$.

The reason for this is that rewriting a double-barred word via $t_{0}$ 's productions $\overline{\bar{a}}_{i} \rightarrow \bar{S}_{i, j}$ is the same as choosing the substitution $\mathcal{T}_{j}$ to be used. The substitution is then performed via the tables $t_{j}$ and $\widetilde{T}_{k, j}$, and when the substitution is performed, the word is again double-barred. We can choose a new substitution and so for th until we finally use the terminal table to reach a terminal word. If the tables to be used in the $\|$ ine of derivation are not chosen according to this scheme, a $\$$-symbol is introduced in the string, and from this it is impossible to reach a terminal word.

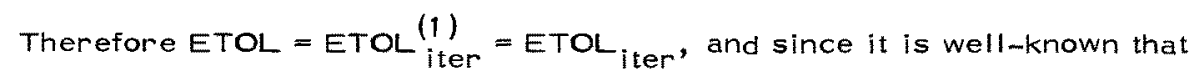
ETOL is a full AFL, we conclude that ETOL is a hyper-AFL. 


\section{Corollary 1}

If $K$ is a family of languages such that $F \subseteq K \subseteq E T O L$ then $K_{i t e r}=E T O L$.

\section{Proof}

$$
E T O L=F_{i t e r} \subseteq K_{i t e r} \subseteq E T O L_{i t e r}=E T O L .
$$

Thus we have for instance proved that:

$$
E T O L=F_{i t e r}=R_{i t e r}=C F_{i t e r}=E O L_{i t e r}=E T O L_{i t e r} .
$$

Since each hyper-AFL is a full AFL and since $E T O L=R_{i t e r}$, we conclude:

\section{Corollary 2}

ETOL is the smallest hyper-AFL.

In $[56]$ it is stated that the family $R_{i t e r}^{(1)}$ is exactly the family of languages accepted by pre-set-pushdown automata, and we are now able to prove:

\section{Corollary 3}

$$
R_{i t e r}^{(1)} \quad F R_{i t e r}=E T O L .
$$

\section{Proof}

By definition $R_{i t e r}^{(1)} \subseteq R_{i t e r}$ and we know that $R_{i t e r}=E T O L$. Furthermore, hyper-AFL's are easily seen to be closed under substitution; in order to prove the corollary, it suffices to prove that $R_{i t e r}^{(1)}$ is not closed under substitution:

Let $L_{1}=\left\{a^{2^{n}} \mid n \geq 0\right\}$ and $L_{2}=\left\{a b^{2^{m}} \mid m \geq 0\right\}$, then obviously $L_{1}, L_{2} \in O L \subseteq E O L=F_{i t e r}^{(1)} \subseteq R_{i t e r}^{(1)}$.

Define the substitution $\mathcal{T}_{\text {by }} \mathcal{T}_{(a)}=L_{2}$. Then $\tau_{\left(L_{1}\right)}$ is the set of all words $a b^{2^{n_{1}}} a b^{2^{n_{2}}} \ldots a b^{2^{n_{k}}}$, where each $n_{i} \geq 0$ and there exists $1 \geq 0$ such that $k=2$. Define the finite substitution $t$ by $t(a)=\{a\}$ and $t(b)=\{\lambda, b\}$ then the proof in [35] of the non-closure of EOL under inverse homomorphism shows that $\mathrm{t}\left(\tau\left(L_{1}\right)\right) \notin E O L=F_{i t e r}^{(1)}$. It is furthermore well-known that EOL is closed under finite substitution, therefore $\mathcal{T}_{\left(L_{1}\right)} \notin F_{i \text { ter }}^{(1)}$. But since infinite regular sets fulfil a pumping lemma, it is obvious that $\mathcal{T}_{\left(L_{1}\right) \notin R} \notin$ iter and the corollary is proved. 
Finally we mention that we have proved the existence of full AFL's $K$ and $\bar{K}$ such that:

$$
K_{i t e r}^{(1)} \subsetneq K_{i t e r}=\bar{K}_{i t e r}^{(1)}=\bar{K}_{i t e r}
$$

and $K \neq K_{i t e r}$ but $\left(K_{i t e r}\right)_{i t e r}=K_{i t e r}$ namely $K=R$ and $\bar{K}=E T O L$. 